Manuscript number: NLM-05-86-revised

\title{
Sleep deprivation impairs object recognition in mice
}

\author{
Svitlana Palchykova, Raphaëlle Winsky-Sommerer, Peter Meerlo \\ Roland Dürr and Irene Tobler*
}

Institute of Pharmacology and Toxicology, University of Zürich, Zürich, Switzerland

${ }^{1}$ Department of Molecular Neurobiology, University of Groningen, Haren, The Netherlands

Running head: Sleep deprivation impairs recognition

Submitted to Neurobiology of Learning and Memory

${ }^{*}$ Corresponding author:

Prof. Irene Tobler Ph.D.

Institute of Pharmacology and Toxicology

University of Zurich

Winterthurerstr. 190

$\mathrm{CH}-8057$ Zurich

Phone: +41(0)44 6355957

Fax: +41 (0)44 6355707

email: tobler@pharma.unizh.ch

Number of figures: 5 figures

Number of pages: 30

Word counts: abstract 280 


\section{Abstract}

Many studies in animals and humans suggest that sleep facilitates learning, memory consolidation and retrieval. Moreover, sleep deprivation (SD) incurred after learning, impaired memory in humans, mice, rats and hamsters. We investigated the importance of sleep and its timing in an object recognition task in OF1 mice subjected to $6 \mathrm{~h} \mathrm{SD}$ either immediately after the acquisition phase (0-6 SD) or $6 \mathrm{~h}$ later (7-12 SD), and in corresponding undisturbed controls. Motor activity was continuously recorded with infra-red sensors.

All groups explored two familiar, previously encountered objects to a similar extent, both at the end of the acquisition phase and $24 \mathrm{~h}$ later during the test phase, indicating intact familiarity detection. During the test phase 0-6 SD mice failed to discriminate between the single novel and the two familiar objects. In contrast, the 7-12 SD group and the two control groups explored the novel object significantly longer than the two familiar objects. Plasma corticosterone levels determined after SD did not differ from time-matched undisturbed controls, but were significantly below the level measured after learning alone. ACTH did not differ between the groups. Therefore, it is unlikely that stress contributed to the memory impairment.

We conclude that the loss of sleep and the activities the mice engaged in during the SD, impaired recognition memory retrieval, when they occurred immediately after acquisition. The delayed SD enabled memory consolidation during the $6 \mathrm{~h}$ when the mice were allowed to sleep, and had no detrimental effect on memory. Neither SD schedule impaired object familiarity processing, suggesting that only specific cognitive abilities were sensitive to the intervention. Sleep may either actively promote memory formation, or 
alternatively, sleep may provide optimal conditions of non-interference for consolidation.

Key words: Sleep deprivation; Object recognition; Familiarity; Stress; Rest; Motor activity 


\section{Introduction}

There is increasing evidence that sleep may be important for learning and memory, whereas a sleep deficit results in performance impairment both in rodents and humans (for review Stickgold, 2005; Walker, 2004). However, the role of sleep in memory formation is complex and appears to depend on the nature of the task (e.g., Graves, Heller, Pack, \& Abel, 2003). Moreover, there are data indicating that sleep should occur within a specific time window following upon the training or acquisition phase in order to facilitate learning effectively (e.g., Pearlman, 1973; Smith \& Butler, 1982; Smith, Tenn, \& Annett, 1991). Thus, $4 \mathrm{~h}$ of REM sleep deprivation immediately following training resulted in learning impairment in the hidden platform of the Morris water maze, but not in the visual platform (Smith \& Rose, 1996). The same duration of REM sleep deprivation performed after acquisition in the eight-arm radial maze task resulted in a deficit of spatial reference memory, whereas working memory was intact (Smith, Conway, \& Rose, 1998). Recent data show that Fisher rats deprived from REM sleep for $4 \mathrm{~h}$ after training, switch from a spatial to a non-spatial strategy to solve a complex associative learning task (Bjorness, Riley, Tysor, \& Poe, 2005). Moreover, $5 \mathrm{~h}$ total sleep deprivation in C57BL/6 mice impaired contextual but not cued fear conditioning, when the SD was scheduled immediately after the acquisition phase (Graves et al., 2003). In contrast, delayed REM sleep deprivation (hours $4-8,8-12$ or $13-24$ after acquisition) or delayed total sleep deprivation (hours 5-10) had no effect on memory (Bjorness et al., 2005; Graves et al., 2003; Smith \& Rose, 1996; Smith et al., 1998). Taken together, these data 
indicate that sleep at the appropriate time, may facilitate and optimize memory formation of certain tasks. On the other hand, the fundamental relevance of sleep in memory processing has been questioned (reviewed in Coenen, 2005; Doyon, Carrier, Simard, Tahar, Morin, Benali, \& Ungerleider, 2005; Korman, Flash, \& Karni, 2005; Schredl, 2005; Siegel, 2005; Vertes, 2004; Vertes, 2005; Vertes \& Siegel, 2005). Important arguments are the specificity of the tasks that seem to profit from sleep, contrasting a global role of sleep in memory consolidation, and the limitations of comparing results obtained in humans and animals.

The one-trial object recognition task, originally developed for rats (Ennaceur \& Delacour, 1988) and applied in several mouse studies (e.g., Dodart, Mathis, \& Ungerer, 1997; Genoux, Haditsch, Knobloch, Michalon, Storm, \& Mansuy, 2002), provides a useful behavioral paradigm to investigate the effects of sleep loss on memory consolidation and retrieval. Animals have to learn to recognize biologically meaningless objects or their spatial location, and should be able to retrieve the object or location information in a complex spatial scene when tested $24 \mathrm{~h}$ later. It was suggested that memories of objects in rodents can be compared to human episodic-like memory (Dere, Silva, \& Huston, 2004; Morris, 2001).

To our knowledge the role of sleep on recognition memory has not been investigated in animals. Therefore, it is unknown whether there is a specific timing of sleep that may be more important for consolidation or retention of recognition memories. To test whether undisturbed sleep occurring immediately after learning is critical and sufficient for consolidation of object recognition, we subjected mice to $6 \mathrm{~h}$ total SD by "gentle handling" 
either immediately (0-6 SD) or $6 \mathrm{~h}$ after the end of an acquisition phase (7-12 SD).

Sleep deprivation has been considered to be stressful for animals (Horne \& McGrath, 1984). Since stress may be a cause for memory impairment, we measured the plasma levels of the stress hormones corticosterone and $\mathrm{ACTH}$ in mice subjected to acquisition alone and in mice subjected to acquisition followed by SD. To evaluate the effectiveness of SD and the effects of learning and SD on rest, motor activity was recorded with infra-red (IR) sensors for several days before the learning task and throughout the experimental days.

\section{Materials and Methods}

\subsection{Animals}

The Cantonal Veterinary Office of Zürich approved all experimental procedures. The experiments were performed in OF1 mice because a detailed analysis of their sleep and the effects of $5 \mathrm{~h}$ sleep deprivation were reported recently (Kopp, Petit, Magistretti, Borbély, \& Tobler, 2002) and OF1 mice have been used in similar object recognition tests (Bour, Little, Dodart, Kelche, \& Mathis, 2004). Adult male outbred OF1 mice ( $n=89)$, weighing $41 \pm 5 \mathrm{~g}(\mathrm{SD})$, were kept individually in Macrolon cages (36 × $20 \times 35 \mathrm{~cm}$ ) with food and water available ad libitum. The animals were maintained on a $12 \mathrm{~h}$ light : $12 \mathrm{~h}$ dark cycle (light onset at 9 A.M.; $~ 30$ lux) at $23^{\circ} \mathrm{C}$ ambient temperature. All 
behavioral tests were performed at the age of $15-16$ wks under dim red lighting conditions (< 5 lux), starting 30 - 105 min before light onset.

\subsection{Activity recordings}

Passive IR activity was recorded continuously throughout the experiment. The sensor mounted above the cage generated a signal (activity counts) in response to spatial movements of the mouse. Counts were integrated over consecutive 1-min epochs and stored on a computer, as described previously (Tobler, Gaus, Deboer, Achermann, Fischer, Rulicke, Moser, Oesch, McBride, \& Manson, 1996; Chronobiology Kit, Stanford Software system, Stanford, CA). The effects of SD or learning on motor activity, rest and the number and duration of rest epochs were investigated based on 1-h or 6-h intervals and compared to baseline 10-day mean values. Two aspects of rest-activity were evaluated: 1 . intensity of activity during activity bouts (total activity counts within an hour divided by the number of 1-min epochs with activity above zero); 2. duration of rest (defined as the number of 1-min epochs with activity counts equal zero).

\subsection{Sleep deprivation procedure}

Mice were subjected to $6 \mathrm{~h}$ SD starting either immediately after the acquisition phase (0-6 SD, $\mathrm{n}=9$ ), coinciding with onset of the 12-h light period, or $6 \mathrm{~h}$ later (7-12 SD, n=9). Animals of the two corresponding time of day control groups ( $n=8-9$ per group) were returned to their home cage immediately after 
acquisition and left undisturbed. The order of testing of SD and control animals was randomized. SD was achieved by "gentle handling" as described previously (Tobler, Deboer, \& Fischer, 1997). During the SD procedure the mice were provided with familiar nesting material (tissues). No novel objects were introduced.

\subsection{Behavioral tasks}

The object and location recognition task comprised an acquisition phase, delay phase, and test phase. All animals were individually familiarized with the experimental context but without objects for 15 min at the end of the 12-h dark period on two consecutive days, immediately preceding the acquisition phase. The floor of a square enclosure (grey plastic, $75 \times 75 \times 37 \mathrm{~cm}$ ) was covered with soiled wood chippings collected from cages of several mice, ensuring saturation with odors of conspecifics. No specific spatial cues were provided within the enclosure. Nine sets of 3-4 identical objects (volume approximately 16 to $18 \mathrm{~cm}^{3}$ ) differing in shape, color and texture and with no biological relevance were kept in the boxes with soiled wood chippings collected from cages of several mice. Objects and their spatial location were randomized among the animals. Video recordings were obtained throughout the acquisition and test phase.

To test memory for a complex scene, four groups of mice (SD 0-6, SD 7-12 and corresponding controls) were given the opportunity to explore a triplet of novel objects for five times 5 min with 15-min intervals between exposures during the acquisition phase at the end of the dark period (Fig. 1). 
Acquisition was followed by a 24-h delay phase during which the mice either experienced a 6-h sleep deficit followed by undisturbed sleep during the remaining 12 - $18 \mathrm{~h}$, or were immediately returned to their home cage and left undisturbed (controls). A 24-h retention period was chosen to avoid a circadian effect on performance (e.g. Chaudhury \& Colwell, 2002) and to provide sufficient time for recovery (Kopp et al., 2002). In the subsequent test phase, the mice were exposed for 5 min to a new triplet of objects: two objects encountered previously in the acquisition phase were replaced by an identical copy (targets), and presented together with a novel object. If recognition memory was intact, the mice were expected to spend more time exploring the novel object (Ennaceur \& Delacour, 1988).

To test memory for the spatial location of objects, a 5th group of mice ( $n=10)$ was allowed to investigate two identical copies of a novel object for five times 5 min with 15-min intervals between exposures during acquisition (Fig. $1 \mathrm{~B}$ ). The objects were placed in two adjacent corners of the square enclosure. In the 5-min test phase $24 \mathrm{~h}$ later, the objects were replaced by two identical copies, one of which was placed in its original location (target), the other in a different corner. The position of the target was counter-balanced among individuals. If the animals perceived the spatial rearrangement of the two familiar objects, they were expected to spend more time exploring the object in the novel location (Ennaceur \& Meliani, 1992). 


\subsection{Plasma ACTH and corticosterone assays}

An additional batch of mice ( $n=24 ; 14-15$ wks old) kept in the same environment and conditions as the experimental groups, was used to collect blood for measurement of stress hormones after learning and SD. Animals were subjected to acquisition of the complex scene learning task, and then subdivided. One group was subjected to $6 \mathrm{~h} \mathrm{SD}$, starting as in the memory experiments, immediately after acquisition and killed at the end of SD ('Acquisition+6hSD'; $n=8$ ), and two groups were killed immediately ('Acquisition'; $\mathrm{n}=8$ ) or $6 \mathrm{~h}$ after the acquisition phase ('Acquisition+6h'; $n=8$ ). Undisturbed controls were kept in individual home-cages in a separate room and killed at the same time of day as the 'Acquisition' (Control 1;n=10) or 'Acquisition+6hSD' group (Control 2; $n=11$ ). An additional group of mice was immobilized for $30 \mathrm{~min}$ at the same time of day as the 'Acquisition+6hSD' group and killed thereafter ('Immobilized'; $n=9$ ).

The mice were decapitated and trunk blood was collected in K2E EDTA K2 tubes (Vacuette ${ }^{\circledR} 2 \mathrm{ml}$, Greiner Bio-One Vacuette Schweiz GmbH) kept on ice and then centrifuged at $2600 \mathrm{~g}$ at $4{ }^{\circ} \mathrm{C}$ for $15 \mathrm{~min}$. The supernatant was stored at $-80{ }^{\circ} \mathrm{C}$ in cryotubes for later analysis. Plasma corticosterone and ACTH concentrations were determined by radioimmunoassay (corticosterone kit, MP Biomedicals, Costa Mesa, USA; ACTH kit, Nichols Institute Diagnostics, Bad Vilbel, Germany). 


\subsection{Groups, variables and statistics}

Interactions with objects were quantified by visual off-line scoring of the video tapes by a trained observer. The exploratory behavior towards objects was quantified as time spent at a distance $<2 \mathrm{~cm}$ and/or contact with the objects. Circling around or sitting on the objects was not considered. For the test phase, an object exploration ratio was calculated. It was defined as the time spent on exploration of the novel object, divided by the time spent exploring the novel object plus the mean time exploring the two familiar target objects. For the acquisition phase, the same ratio was calculated for the object occupying the same location as the novel object during the test phase. A ratio of 0.5 indicated similar exploration of the novel and familiar target objects, corresponding to chance; a ratio $>0.5$ indicated a higher exploration of the novel object.

A two-way ANOVA with between subject factor 'group' and within subject factor 'object', 'phase (acquisition, test)' or 'session $(1,5)$ ' was used to analyze behavioral data and one-way ANOVA (factor 'group') and KruskalWallis test (when not normally distributed; normality was tested with the procedure Univariate; SAS) was used for plasma corticosterone levels (after decimal logarithm transformation), and plasma ACTH levels. Activity and rest were analyzed with two-way ANOVA for repeated measures, within subject factor 'day (baseline vs. acquisition)' and 'interval (1- or 6-h values)'. When significance was reached, post-hoc testing was performed with the Tukey test, unpaired or paired t-tests for between and within group comparisons, respectively, and non-parametric Kruskal-Wallis and Wilcoxon signed-rank 
tests for samples that were not normally distributed. For object exploration, the mean for the two target objects was used, after ensuring by statistical testing that exploration at study or test did not differ significantly.

\section{Results}

\subsection{Object recognition in the complex scene in OF1 mice}

During the acquisition phase, the two control groups explored each of the three objects to the same extent ['object' $F(2,30)=0.77$, 'group' $F(1,15)=1.63$, 'object $x$ group' $F(2.30)=0.01$ ] and displayed a reduction in their exploration [Fig. 2 A; 'session: 1 vs 5' $F(1,15)=8.67, p<.006$, 'group' $F(1,15)=0.13$, 'group x session' $F(1,15)=0.04]$.

During the test phase (Fig. 2 B), both control groups spent more time exploring the novel object compared to the two familiar objects $[p<.05$ Tukey test after two-way ANOVA factor 'object' $F(1,15)=12.33$ was $p<.001]$. In addition, there was a significant group effect ['group' $F(1,15)=6.18, p<.019$ ] Thus, total object exploration was lower in controls of the 7-12 SD than those of the 0-6 SD ( $p<.05$, Tukey test). However, the ANOVA interaction 'group $x$ objects' was n.s. $[F(1,15)=2.39]$, indicating that the controls did not differ in their discrimination performance. The analysis of the exploration ratio confirmed this finding (Fig. 2 C). Both control groups showed a similar increase in the exploration ratio from acquisition to test $[p<.05$ Tukey, 'phase' $F(1,15)=16.33, p<.0003$, 'group' $F(1,15)=0.50$, 'group $x$ phase' $F(1,15)=0.22]$. 


\subsection{A 6-h sleep deprivation immediately after the acquisition phase led to object recognition impairment in the complex scene}

During the acquisition phase, each of the three objects was explored to the same extent ['object' $F(2,32)=0.15]$, by both groups ['group: control, SD' $F(1,16)=0.86$, 'group $x$ object' $F(2,32)=0.74]$. Moreover, the time the animals spent exploring the objects decreased progressively in both groups (Fig. $2 \mathrm{~A}$ left). The comparison of time spent on the two (familiar) target objects during the test phase compared to time spent on the same objects at the end of the acquisition phase did not differ within either group ['phase: last 5 min of acquisition vs. test' $F(1,16)=1.02]$. This result indicates that in both groups familiarity detection was intact ['group' $F(1,16)=0.28$ and 'group $x$ phase' $F(1,16)=1.17]$.

In the test phase, a significant detrimental effect of SD was evident by the failure of the sleep deprived mice to discriminate between the familiar and novel objects. Thus, the SD group explored the novel object to the same extent as the two familiar targets (Fig. 2 B left), whereas the control group spent significantly more time exploring the novel object. Also the group comparison showed an impairment in the SD group, by their significantly lower exploration of the novel object compared to the control group, while both groups explored the familiar objects to a similar extent [Fig. 2 B left; duration of exploration: 'group' $F(1,16)=8.26, p<.007$; 'group $x$ object: novel, mean or two familiar' $F(1,16)=5.43, p<.03]$.

The exploration ratio confirmed the failure of the SD mice to discriminate between the novel and familiar objects: in the SD mice the ratio 
did not differ between acquisition and test (Fig. $2 \mathrm{C}$ left), while it increased significantly in controls. The groups did not differ during acquisition (Fig. 2 C left, black bars), while at test (white bars) the ratio was significantly lower in the SD group than in the controls ['phase' $F(1,16)=9.49, p<.0042$, 'group $x$ phase' $F(1,16)=3.47, p<.0718]$.

In summary, SD during the first $6 \mathrm{~h}$ after exposure to three objects in the acquisition phase impaired the ability to discriminate between two objects encountered previously and a novel one.

\subsection{No effect of a delayed sleep deprivation}

As in the previous experiment, during the acquisition phase each of the three objects was explored to the same extent ['object' $F(2,30)=0.57$ ] by both groups ['group: control, SD' $F(1,15)=0.12$, 'group $x$ object' $F(2,30)=0.19]$. Again, there was a similar progressive reduction in the time the animals spent exploring the objects (Fig. 2 A right). The time spent on the two (familiar) target objects during the test phase was similar to time on the same objects at the end of the acquisition ['phase' $F(1,15)=0.84$ ]. This result was similar between the groups ['group' $F(1,15)=2.49$, 'group $x$ phase' $F(1.15)=0$ ], indicating intact familiarity detection, despite the SD.

In contrast to the 0-6 SD experiment, there was no effect of SD in the 7-12 SD paradigm during the test phase. Thus, both control and sleepdeprived mice spent significantly more time exploring the novel object compared to the two familiar objects [Fig. 2 B right; $p<.05$ Tukey test, 'object' 
$F(1,15)=4.62, p<.039$ ]. No group difference occurred ['group' $F(1,15)=2.76$, 'group $\mathrm{x}$ object' $F(1,15)=0.26]$.

The comparison of the mean exploration ratio within the acquisition phase and test showed a significant increase from acquisition to test in both groups [Fig. $2 \mathrm{C}$ right; $p<.05$, Tukey, 'phase' $F(1,15)=13.86, p=.0008$ ]. There was no group difference either within the acquisition or test phases ['group' $F(1,15)=0.15$, 'group $x$ phase' $F(1,15)=0.01]$.

In summary, the 6-h SD experienced $6 \mathrm{~h}$ after the acquisition phase did not affect the ability to discriminate between a novel and two previously encountered objects.

\subsection{Memory for spatial location of object}

As was expected, the mice explored the two identical novel objects to the same extent during the acquisition phase (total exploration during entire acquisition phase: $75.9 \mathrm{~s} \pm 28.1$ (SE) and $79.8 \mathrm{~s} \pm 21.9$, n.s., Wilcoxon signed-ranks test). During the test phase, in contrast to the expectation that the object in the novel location would be explored more than the one in the same location as previously, there was no difference in exploration of two duplicates of the original objects, when one of them occupied a novel location (5-min test phase: $19.5 \pm 6.0 \mathrm{~s}$ and $20.9 \pm 7.9 \mathrm{~s}$, n.s., Wilcoxon). Thus, even the undisturbed control group was unable to detect the spatial rearrangement of two already seen objects under this training paradigm after the 24-h delay. Therefore, the effect of SD was not evaluated in this task. 


\subsection{Activity and rest}

The loss of rest during the 6-h SD interval was larger in the delayed SD paradigm compared to the immediate $S D[t(16)=12.39, p<.0001$, unpaired $t$ test; number of rest epochs during the SD vs. the corresponding baseline: 0-6 SD, $261.9 \pm 3.4 \mathrm{~min}, 61.7 \pm 6.9 \mathrm{~min} ; 7-12 \mathrm{SD}, 335.8 \pm 1.5 \mathrm{~min}, 42.8 \pm 7.4 \mathrm{~min}$, $p<.0001$, paired t-test after 'day $\times$ 6-h interval' $F(3,48)=78.77$ and $F(3,48)=92.37, p<.0001$, respectively]. During recovery after 0-6 SD, changes in intensity of activity were minor and the amount of rest was unchanged (Fig. 3). In contrast, after 7-12 SD, immediate and prolonged changes occurred: activity was reduced during almost the entire subsequent dark period, while rest was significantly increased for $3 \mathrm{~h}$ (Fig. 3). In the control groups, neither activity nor rest differed significantly between baseline and the experimental days (0-6 SD control: activity, 'day' $F(1,16)=0.0$, 'day $x$ 1-h interval' $F(23,32)=0.99$, rest, 'day' $F(1,16)=0.21$, 'day $\times 1$-h interval' $F(23,32)=0.91 ; 7-$ 12 SD control: activity, 'day' $F(1,14)=0.08$, 'day x 1-h interval' $F(23,28)=0.70$, rest, 'day' $F(1,14)=0.28$, 'day x 1 -h interval' $F(23,28)=0.93)$.

Analysis of rest episode duration during recovery revealed that the immediate SD (SD 0-6) led to a significant increase in the number of long rest episodes in the first 6-h interval after SD (Fig. 4 left). After the delayed SD (SD 7-12), the number of short rest episodes decreased significantly (Fig. 4 right). The immediate SD still exerted a significant effect in the second 6-h recovery interval, where short rest episodes were significantly decreased.

During the last $90 \mathrm{~min}$ of the baseline dark period, corresponding to the acquisition phase on experimental days, rest comprised $61.7 \pm 1.4$ min (mean 
of 10 days). The acquisition phase was followed by a significant consolidation of rest. Thus, in the 6-h interval following acquisition the number of rest episodes longer than $16 \mathrm{~min}$ was increased from $1.60 \pm 0.11$ to $2.19 \pm 0.22$ $(t=2.72, p<.05$, paired t-test $)$ at the cost of short rest episodes $(<5 \mathrm{~min}$, from $14.61 \pm 1.36$ to $11.27 \pm 1.00, t=3.19, p<.005)(n=26$; pooled values of the three groups undisturbed immediately after acquisition: two controls and 7-12 SD).

\subsection{Stress hormones}

Corticosterone levels at the end of the acquisition phase ('Acquisition') were significantly above the values of the undisturbed time-matched 'Control 1' (Fig. 6 left; $p<.0001$, unpaired t-test). SD following acquisition induced a mild but significant increase of plasma corticosterone above the levels of timematched mice subjected to the acquisition phase only $[p<.05$, Tukey after 'group (3-6)' $F(3,32)=45.49, p<.0001]$. However, neither of these two groups differed from the undisturbed time-matched controls. Also, the corticosterone level at the end of SD following the acquisition phase was significantly lower than immediately after acquisition alone $(p<.05$, Tukey). For comparison, immobilization stress led to a major increase of plasma corticosterone above the amount encountered in all other groups ( $p<.05$, Tukey), with the exception of the level immediately following the acquisition phase.

The levels of plasma ACTH attained after immobilization were significantly above the values of the other groups (Fig. 5 right; $p<.05$, Tukey). There were no further differences between the groups. 


\section{Discussion}

A period of sleep deprivation following immediately upon an acquisition phase caused a significant retrieval detriment in a complex spatial scene paradigm $24 \mathrm{~h}$ later. This deficit in object discrimination was restricted to the group of mice subjected to SD during the first $6 \mathrm{~h}$ following acquisition, since both the controls that were allowed to sleep, as well as the mice subjected to a similar but delayed SD had no such deficit (Fig. 2 C). Apparently, only the early intervention interfered with the process of memory consolidation necessary for optimal performance, while $6 \mathrm{~h}$ of undisturbed sleep following immediately upon the acquisition phase were sufficient for memory consolidation. Our results are consistent with the negative or modulating effects on performance of SD timed immediately after acquisition in contextual fear conditioning in C57BL/6 mice (Graves et al., 2003), in an object recognition task in hamsters (Palchykova, Crestani, Meerlo, \& Tobler, 2005) and in an associative learning task in rats (Bjorness et al., 2005). Taken together the results confirm that the timing of sleep is crucial for its beneficial effect on memory consolidation and retrieval.

Many studies have suggested that sleep-specific physiological processes may actively facilitate learning. Thus, correlation analysis of neuronal activity recorded in freely moving rodents shows that distinct firing patterns of cells during waking tend to recur during sleep (e.g., Dave \& Margoliash, 2000; Hirase, Leinekugel, Czurko, Csicsvari, \& Buzsaki, 2001; Louie \& Wilson, 2001; Pavlides \& Winson, 1989; Wilson \& McNaughton, 1994). Several neuronal changes during sleep have been related to learning. 
These include hippocampal sharp waves (Nadasdy, Hirase, Czurko, Csicsvari, \& Buzsaki, 1999), phasic pontine-waves (Datta, 2000), phase shift of hippocampal firing activity (Poe, Nitz, McNaughton, \& Barnes, 2000) and synaptic downscaling (Tononi \& Cirelli, 2001). In addition, genes which are essential for synaptic plasticity and memory formation (Davis, Bozon, \& Laroche, 2003; Jones, Errington, French, Fine, Bliss, Garel, Charnay, Bozon, Laroche, \& Davis, 2001) are regulated during sleep (Cirelli, Gutierrez, \& Tononi, 2004; Ribeiro, Goyal, Mello, \& Pavlides, 1999). In contrast to a direct involvement of sleep in memory consolidation, several studies suggest that sleep may facilitate memory consolidation by preventing waking interference. Motor activity, sensory input or additional learning occurring during waking can be sources of interference with the process of memory consolidation. Thus, there is evidence in humans that training of an additional task during the retention phase (Walker, Brakefield, Hobson, \& Stickgold, 2003) as well as repeated testing of the same task (Mednick, Nakayama, Cantero, Atienza, Levin, Pathak, \& Stickgold, 2002) can reverse a previously consolidated memory into a labile state, whereas restful waking can facilitate learning and provide similar benefits as sleep (Gottselig, Hofer-Tinguely, Borbély, Regel, Landolt, Retey, \& Achermann, 2004). During the SD, the mice were activated by introducing nesting material (tissues) and by other subtle stimulations. These motor and sensory stimuli could have interfered with memory consolidation.

All groups displayed a progressive reduction of object exploration during acquisition. Such habituation to objects during the acquisition phase has been shown previously in a similar paradigm in mice (Genoux et al., 
2002). When the animals encountered the objects again in the test phase, it was evident that they all recognized the target objects as familiar because they did not increase the exploration of the familiar object (Fig. 2 A and B). Therefore, neither controls nor the SD groups had a memory consolidation problem. Despite intact memory consolidation, the 0-6 SD group treated the novel object as familiar, thus failing to recognize the novelty. This result suggests that the objects were remembered as a general category, indicating that mice were capable of learning in one trial an absolute class concept 'object' and to form a familiarity-based memory for other objects belonging to the same class. Therefore, in contrast to the deficit in object discrimination, neither SD paradigm interfered with the formation of familiarity-based memory for a discrete object. In summary, the cognitive deficit induced by SD was related to a specific process involved in recognition memory.

It is unlikely that lack of motivation was responsible for the deficit in object discrimination observed in the early SD group, because the 24-h retention interval and the 18 and $12 \mathrm{~h}$ recovery from SD in the early and late SD groups, respectively, allowed sufficient time to recover from the sleep deprivation, as shown by Kopp et al. (2002) and supported by the time course of rest and activity after the SD (Fig. 3). Despite the larger loss of sleep due to the circadian timing of the SD in the 7-12 SD group, memory was impaired only in the 0-6 SD group. Therefore, it was a memory deficiency and not lack of motivation that was impaired by the early SD. This interpretation is supported by the slightly longer time spent exploring the objects upon test in this early SD group compared to the 7-12 SD group. 
A stable memory for a whole scene requires knowledge of the specific features of objects, but also of their spatio-temporal relationships (Gaffan, Healey, \& Eacott, 2004). Hence, inability to retrieve spatio-temporal information might have been responsible for the performance deficit induced by SD. One can hypothesize that the $0-6 \mathrm{~h}$ SD animals might have encoded the new scene encountered at test as a novel one, despite the presence of the (familiar) targets. To evaluate this possibility, we tested the ability of the mice to detect a spatial rearrangement of familiar objects. The mice failed to discriminate between the objects in the familiar and novel location $24 \mathrm{~h}$ later, precluding the assessment of SD effects. This incapacity is consistent with data showing that C57BL/6 mice and several rat strains (DA, Sprague-Dawley and Lister) tested in the object location task successfully discriminated between the familiar and novel object location after a delay interval of up to 6 h, while others (DBA/2 mice and Wistar rats) did not (Dix \& Aggleton, 1999; Ennaceur, Michalikova, Bradford, \& Ahmed, 2005; Hotte, Naudon, \& Jay, 2005; Lee, Hunsaker, \& Kesner, 2005; Roullet, Mele, \& Ammassari-Teule, 1997). This failure in discrimination between the familiar and novel location of the objects can be related to either impaired memory for the object per se or inability to use spatial information after the relatively long delay of $24 \mathrm{~h}$. Because the mice were able to detect object familiarity in the complex scene, it is likely that retrieval of spatial memory was hampered.

It is well known that sleep-wake or rest-activity behavior varies across $24 \mathrm{~h}$ in rodents. Thus, the OF1 strain exhibits higher levels of activity during the first half of the light period than in the second $6 \mathrm{~h}$ (Kopp et al., 2002). Consistent with this finding, the delayed SD induced a larger loss of rest than 
the immediate one, when it was compared to the amount of rest the animals obtained during the corresponding baseline interval. Despite this difference in loss of rest between the SD groups, it was the 7-12 SD group that showed no SD impairment on memory. It is well known that $6 \mathrm{~h} \mathrm{SD}$ induce a prominent increase in SWA in NREM sleep during recovery (e.g., Tobler, 2005). The significant increase in rest consolidation during recovery after both SD paradigms could be reflecting an increase in sleep intensity (Fig. 4). A consolidation of rest occurred also after the acquisition phase in the mice that were left undisturbed. This finding may be a homeostatic response to sleep loss during the acquisition phase as well as a consequence of the learning paradigm. The rest consolidation following upon learning supports the notion that early sleep (or rest) may provide optimal conditions for memory consolidation.

Stress may be a confounding factor of SD procedures (Horne \& McGrath, 1984). Both acute stress and a rise in the levels of stress hormones can modify memory formation and processing at retrieval, depending on the stressor and the magnitude of hormone increase (for review Kim \& Diamond, 2002; Wolf, 2003). In our study, exposure to novel objects during the acquisition phase induced a rise of corticosterone, which gradually returned to baseline during the following $6 \mathrm{~h}$, even when animals were sleep deprived (Fig. 5). Importantly, despite slightly higher plasma levels of corticosterone in animals sleep-deprived immediately after acquisition compared to mice that were left undisturbed, both levels did not differ from undisturbed time-matched controls. Moreover, plasma levels of ACTH and corticosterone in the sleepdeprived animals were much lower than those of the immobilized mice. We 
conclude that it is unlikely that stress contributed to the memory deficit induced by SD. Moreover, the memory deficit was selective, since familiarity processing was intact. Hence, any difference in performance between the groups can be attributed to the early intervention, the only factor varying consistently across groups.

In conclusion, the loss of sleep or the additional activities of the mice during the SD impaired recognition memory retrieval, when they were incurred immediately after acquisition, whereas the delayed SD that had allowed $6 \mathrm{~h}$ of undisturbed sleep had no such effect. Neither SD schedule impaired object familiarity processing, suggesting a selectivity of the cognitive impairment induced by the intervention.

\section{Acknowledgment}

We thank members of the group for their help during the SD. This work was supported by the Swiss National Science Foundation grants nr. 3100053005.97/2 and 3100A0-100567/1 and EU grant STREP-518189. 


\section{References}

Bjorness, T. E., Riley, B. T., Tysor, M. K., \& Poe, G. R. (2005). REM restriction persistently alters strategy used to solve a spatial task. Learn Mem, 12, 352359.

Bour, A., Little, S., Dodart, J. C., Kelche, C., \& Mathis, C. (2004). A secreted form of the beta-amyloid precursor protein (sAPP695) improves spatial recognition memory in OF1 mice. Neurobiol Learn Mem, 81, 27-38.

Chaudhury, D., \& Colwell, C. S. (2002). Circadian modulation of learning and memory in fear-conditioned mice. Behav Brain Res, 133, 95-108.

Cirelli, C., Gutierrez, C. M., \& Tononi, G. (2004). Extensive and divergent effects of sleep and wakefulness on brain gene expression. Neuron, 41, 35-43.

Coenen, A. (2005). Where is the classic interference theory for sleep and memory? Behavioral and Brain Sciences, 28, 67-68.

Datta, S. (2000). Avoidance task training potentiates phasic pontine-wave density in the rat: a mechanism for sleep-dependent plasticity. J. Neurosci., 20, 86078613.

Dave, A. S., \& Margoliash, D. (2000). Song replay during sleep and computational rules for sensorimotor vocal learning. Science, 290, 812-816.

Davis, S., Bozon, B., \& Laroche, S. (2003). How necessary is the activation of the immediate early gene zif268 in synaptic plasticity and learning? Behav Brain Res, 142, 17-30.

Dere, E., Silva, M. A. D., \& Huston, J. P. (2004). Higher order memories for objects encountered in different spatio-temporaral contexts in mice: Evidence for episodic memory. Reviews in the Neurosciences, 15, 231-240.

Dix, S. L., \& Aggleton, J. P. (1999). Extending the spontaneous preference test of recognition: evidence of object-location and object-context recognition. Behav Brain Res, 99, 191-200.

Dodart, J. C., Mathis, C., \& Ungerer, A. (1997). Scopolamine-induced deficits in a two-trial object recognition task in mice. Neuroreport, 8, 1173-1178.

Doyon, J., Carrier, J., Simard, A., Tahar, A. H., Morin, A., Benali, H., \& Ungerleider, L. G. (2005). Motor memory: Consolidation-based enhancement effect revisited. Behavioral and Brain Sciences, 28, 68-69.

Ennaceur, A., \& Delacour, J. (1988). A new one-trial test for neurobiological studies of memory in rats. 1: Behavioral data. Behav. Brain Res., 31, 47-59.

Ennaceur, A., \& Meliani, K. (1992). A new one-trial test for neurobiological studies of memory in rats. III. Spatial vs. non-spatial working memory. Behav. Brain Res., 51, 83-92.

Ennaceur, A., Michalikova, S., Bradford, A., \& Ahmed, S. (2005). Detailed analysis of the behavior of Lister and Wistar rats in anxiety, object recognition and object location tasks. Behav Brain Res, 159, 247-266.

Gaffan, E. A., Healey, A. N., \& Eacott, M. J. (2004). Objects and positions in visual scenes: effects of perirhinal and postrhinal cortex lesions in the rat. Behav. Neurosci., 118, 992-1010.

Genoux, D., Haditsch, U., Knobloch, M., Michalon, A., Storm, D., \& Mansuy, I. M. (2002). Protein phosphatase 1 is a molecular constraint on learning and memory. Nature, 418, 970-975.

Gottselig, J. M., Hofer-Tinguely, G., Borbély, A. A., Regel, S. J., Landolt, H. P., Retey, J. V., \& Achermann, P. (2004). Sleep and rest facilitate auditory learning. Neuroscience, 127, 557-561. 
Graves, L. A., Heller, E. A., Pack, A. I., \& Abel, T. (2003). Sleep deprivation selectively impairs memory consolidation for contextual fear conditioning. Learn. Mem., 10, 168-176.

Hirase, H., Leinekugel, X., Czurko, A., Csicsvari, J., \& Buzsaki, G. (2001). Firing rates of hippocampal neurons are preserved during subsequent sleep episodes and modified by novel awake experience. Proc Natl Acad Sci U S A, 98, 93869390.

Horne, J. A., \& McGrath, M. J. (1984). The consolidation hypothesis for REM sleep function: stress and other confounding factors--a review. Biol Psychol, 18, 165-184.

Hotte, M., Naudon, L., \& Jay, T. M. (2005). Modulation of recognition and temporal order memory retrieval by dopamine $\mathrm{D}(1)$ receptor in rats. Neurobiol Learn Mem, 84, 85-92.

Jones, M. W., Errington, M. L., French, P. J., Fine, A., Bliss, T. V., Garel, S., Charnay, P., Bozon, B., Laroche, S., \& Davis, S. (2001). A requirement for the immediate early gene Zif268 in the expression of late LTP and long-term memories. Nat Neurosci, 4, 289-296.

Kim, J. J., \& Diamond, D. M. (2002). The stressed hippocampus, synaptic plasticity and lost memories. Nat. Rev. Neurosci., 3, 453-462.

Kopp, C., Petit, J. M., Magistretti, P., Borbély, A. A., \& Tobler, I. (2002).

Comparison of the effects of modafinil and sleep deprivation on sleep and cortical EEG spectra in mice. Neuropharmacology, 43, 110-118.

Korman, M., Flash, T., \& Karni, A. (2005). Resistance to interference and the emergence of delayed gains in newly acquired procedural memories: Synaptic and system consolidation? Behavioral and Brain Sciences, 28, 74-75.

Lee, I., Hunsaker, M. R., \& Kesner, R. P. (2005). The role of hippocampal subregions in detecting spatial novelty. Behav Neurosci, 119, 145-153.

Louie, K., \& Wilson, M. A. (2001). Temporally structured replay of awake hippocampal ensemble activity during rapid eye movement sleep. Neuron, 29, 145-156.

Mednick, S. C., Nakayama, K., Cantero, J. L., Atienza, M., Levin, A. A., Pathak, N., \& Stickgold, R. (2002). The restorative effect of naps on perceptual deterioration. Nat Neurosci, 5, 677-681.

Morris, R. G. (2001). Episodic-like memory in animals: psychological criteria, neural mechanisms and the value of episodic-like tasks to investigate animal models of neurodegenerative disease. Philos Trans R Soc Lond B Biol Sci, 356, 14531465 .

Nadasdy, Z., Hirase, H., Czurko, A., Csicsvari, J., \& Buzsaki, G. (1999). Replay and time compression of recurring spike sequences in the hippocampus. $J$ Neurosci, 19, 9497-9507.

Palchykova, S., Crestani, F., Meerlo, P., \& Tobler, I. (2005). Sleep deprivation and daily torpor impair object recognition in Djungarian hamsters. Physiology \& Behavior, in press.

Pavlides, C., \& Winson, J. (1989). Influences of hippocampal place cell firing in the awake state on the activity of these cells during subsequent sleep episodes. $J$ Neurosci, 9, 2907-2918.

Pearlman, C. (1973). REM sleep deprivation impairs latent extinction in rats. Physiol Behav, 11, 233-237. 
Poe, G. R., Nitz, D. A., McNaughton, B. L., \& Barnes, C. A. (2000). Experiencedependent phase-reversal of hippocampal neuron firing during REM sleep. Brain Res, 855, 176-180.

Ribeiro, S., Goyal, V., Mello, C. V., \& Pavlides, C. (1999). Brain gene expression during REM sleep depends on prior waking experience. Learn Mem, 6, 500508.

Roullet, P., Mele, A., \& Ammassari-Teule, M. (1997). Ibotenic lesions of the nucleus accumbens promote reactivity to spatial novelty in nonreactive DBA mice: implications for neural mechanisms subserving spatial information encoding. Behav Neurosci, 111, 976-984.

Schredl, M. (2005). REM sleep, dreaming, and procedural memory. Behavioral and Brain Sciences, 28, 80-81.

Siegel, J. M. (2005). The incredible, shrinking sleep-learning connection. Behavioral and Brain Sciences, 28, 82-83.

Smith, C., \& Butler, S. (1982). Paradoxical sleep at selective times following training is necessary for learning. Physiol Behav, 29, 469-473.

Smith, C., \& Rose, G. M. (1996). Evidence for a paradoxical sleep window for place learning in the Morris water maze. Physiol. Behav., 59, 93-97.

Smith, C., Tenn, C., \& Annett, R. (1991). Some biochemical and behavioural aspects of the paradoxical sleep window. Can J Psychol, 45, 115-124.

Smith, C. T., Conway, J. M., \& Rose, G. M. (1998). Brief paradoxical sleep deprivation impairs reference, but not working, memory in the radial arm maze task. Neurobiol. Learn. Mem., 69, 211-217.

Stickgold, R. (2005). Sleep-dependent memory consolidation. Nature, 437, 12721278.

Tobler, I. (2005). Phylogeny of Sleep Regulation. In M. H. Kryger, T. Roth, \& W. C. Dement (Eds.), Principles and practice of sleep medicine (pp. 72-81). Philadelphia, PA: Elsevier Saunders.

Tobler, I., Deboer, T., \& Fischer, M. (1997). Sleep and sleep regulation in normal and prion protein-deficient mice. J. Neurosci., 17, 1869-1879.

Tobler, I., Gaus, S. E., Deboer, T., Achermann, P., Fischer, M., Rulicke, T., Moser, M., Oesch, B., McBride, P. A., \& Manson, J. C. (1996). Altered circadian activity rhythms and sleep in mice devoid of prion protein. Nature, 380, 639642.

Tononi, G., \& Cirelli, C. (2001). Some considerations on sleep and neural plasticity. Arch. Ital. Biol., 139, 221-241.

Vertes, R. P. (2004). Memory consolidation in sleep; dream or reality. Neuron, 44, 135-148.

Vertes, R. P. (2005). Sleep is for rest, waking consciousness is for learning and memory - of any kind. Behavioral and Brain Sciences, 28, 86-87.

Vertes, R. P., \& Siegel, J. M. (2005). Time for the sleep community to take a critical look at the purported role of sleep in memory processing. Sleep, 28, 12281229; discussion 1230-1233.

Walker, M. P. (2004). Issues surrounding sleep-dependent memory consolidation and plasticity. Cell Mol Life Sci, 61, 3009-3015.

Walker, M. P., Brakefield, T., Hobson, J. A., \& Stickgold, R. (2003). Dissociable stages of human memory consolidation and reconsolidation. Nature, 425, 616620.

Wilson, M. A., \& McNaughton, B. L. (1994). Reactivation of hippocampal ensemble memories during sleep. Science, 265, 676-679. 
Wolf, O. T. (2003). HPA axis and memory. Best Pract Res Clin Endocrinol Metab, $17,287-299$. 


\section{Figure legends}

\section{Figure 1}

Photo and scheme of the complex spatial scene version of the object recognition task. During the acquisition phase mice were exposed to a triplet of novel objects for five times 5 min with 15-min intervals between exposures (top) or two identical copies of a novel object placed in two adjacent corners of the enclosure (bottom). Acquisition was followed by a 24-h delay phase. During the test phase, the mice were exposed for 5 min to a new triplet of objects: two objects encountered previously during acquisition were replaced by an identical copy and presented together with a novel object (top) or the objects were replaced by two identical copies, one of which was placed in its original location, the other in a different corner (bottom).

\section{Figure 2}

Effects of $6 \mathrm{~h}$ sleep deprivation (SD) performed either immediately after acquisition (0-6 SD; left) or $6 \mathrm{~h}$ later (7-12 SD; right) on object recognition. Left: (A) Time course of object exploration (mean duration in seconds per object) during the acquisition phase in the 0-6 SD and control mice ['session' $F(1,16)=4.67, p<.04$; 'group' $F(1,16)=0.13$ and 'group $x$ session' $F(1,16)=2.05$; $n=9$ per group]. (B) Time spent exploring the familiar objects (mean of two objects) and a single novel object during the test phase. Control mice explored the novel object longer than the familiar objects $\left({ }^{*} p<.05\right.$, paired $t$ test), whereas 0-6 SD mice did not. The 0-6 SD group explored the novel object less than the controls ( ${ }^{\#} p<.05$, Kruskal-Wallis). (C) The exploration ratio during the acquisition (mean per session) and test phase in the two groups 
(acquisition vs. test: ${ }^{*} p<.05$, paired t-test; 0-6 SD vs. controls: ${ }^{*} p<.05$, unpaired $t$-test).

Right: (A) Time course of object exploration during the acquisition phase in 7-12 SD $(n=9)$ and control mice $(n=8)$ ['session' $F(1,15)=14.22$, $p<.0007$, 'group' $F(1,15)=0.05$ and 'group $x$ session' $F(1.15)=1.62]$. (B) Time spent exploring the familiar objects and a single novel object during the test phase (controls, $t=2.57,{ }^{*} p<.05 ; 7-12 \mathrm{SD}, t=1.97,{ }^{\circ} p=.08$ ). (C) The exploration ratio during the acquisition and test phase in the two groups (acquisition vs. test: controls, $\left.t=1.94,{ }^{\circ} p=.09 ; 7-12 \mathrm{SD}, t=2.76,{ }^{*} p<.05\right)$. Mean values $\pm \mathrm{SE}$.

\section{Figure 3}

Top: Time course of infra-red (IR) activity intensity (1-h values, mean \pm SE, arbitrary units, IR-counts/number of 1-min epochs with counts $>0$ ) during baseline (average of 10 undisturbed days) and the sleep deprivation (SD) day (left: 0-6 SD ['day x interval' $F(23,368)=1.99, p<.0048$ ]; right: delayed 7-12 SD ['day $x$ interval' $F(23,368)=9.30, p<.0001]$; $n=9$ per group). Bottom: Rest (number of epochs with activity=0 per hour) (left: 0-6 SD ['day $x$ interval' $F(23,368)=15.0, p<.0001]$; right: $7-12$ SD ['day $x$ interval' $F(23,368)=23.76$, $p<.0001])$. Triangles: SD vs. baseline, $p<.05$, paired $t$-test.

\section{Figure 4}

Number of rest episodes (duration in min: $<5,6-15,16-90$ and $>90$ ) after sleep deprivation (SD) performed immediately after acquisition (Left panels) or $6 \mathrm{~h}$ later (delayed SD; Right panels) and during corresponding baseline intervals (average of 10 undisturbed days; $n=9$ per group). Mean values for $6-\mathrm{h}$ 
intervals \pm SE. SD vs. baseline: ${ }^{*} p<.05,{ }^{* *} p<.005 ; 0-6 \mathrm{SD}(6-15 \mathrm{~min}$, first $6 \mathrm{~h})$, 7-12 SD (>90 min, first and second $6 \mathrm{~h}$ ), $p=.06$, paired $t$-test.

\section{Figure 5}

Plasma levels of corticosterone $(\mathrm{ng} / \mathrm{ml})$ and ACTH $(\mathrm{pg} / \mathrm{ml})$ in mice subjected to the acquisition phase of the object recognition task and $6 \mathrm{~h}$ sleep deprivation (SD). Mice were sacrificed at two time points: 10 and $16 \mathrm{~h}$ (Light from 10:00-22:00 h). Acquisition alone ('Acquisition'; $n=8$ ) induced a large increase in corticosterone which recovered during the subsequent $6 \mathrm{~h}$ in mice left undisturbed after the acquisition phase ('Acquisition $+6 \mathrm{~h}$ '; $n=8$ ), but also in the mice sleep deprived ('Acquisition +6hSD'; $n=8$ ). Control 1 and 2: timematched undisturbed groups ( $n=10-11)$; 'Immobilized': mice immobilized for $30 \min (n=9)$. Mean values \pm SE. Triangles above bars indicate significant differences between the bar and other groups (legend on the side): $p<0.05$, Tukey test. Orientation of triangles indicates the direction of deviation. 
Figure 1

Complex spatial scene

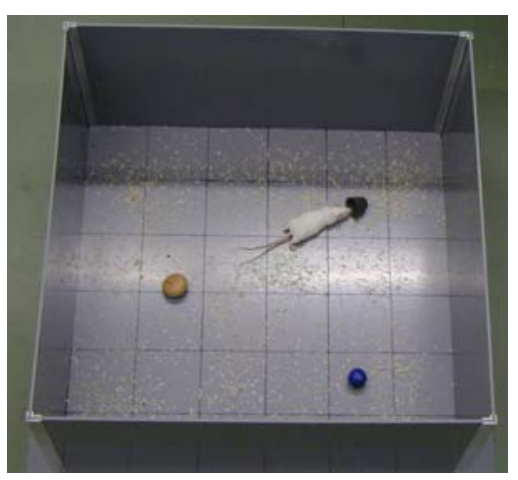

Acquisition

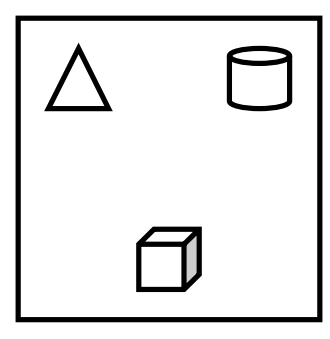

Delay

Test

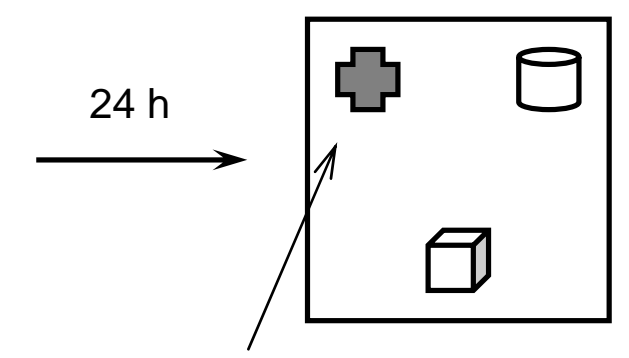

Novel object

Spatial location of objects

Acquisition

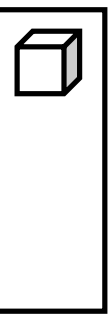

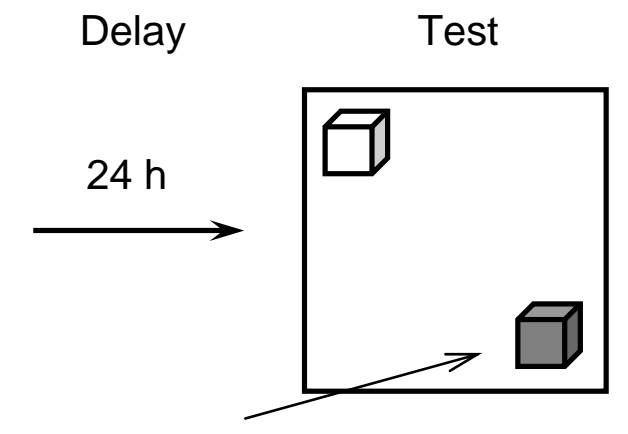

Novel Location 


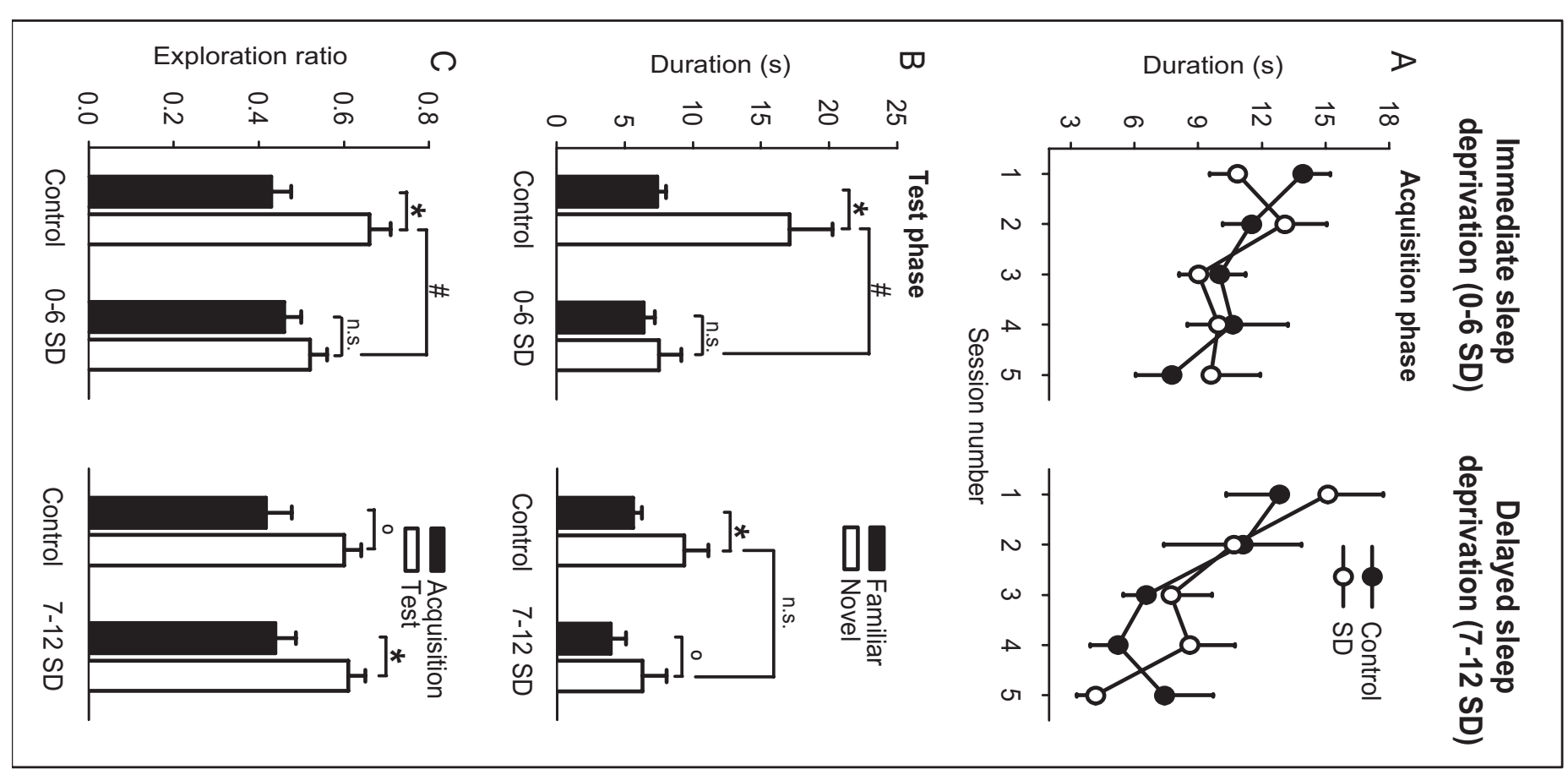


Figure 3

Immediate sleep deprivation
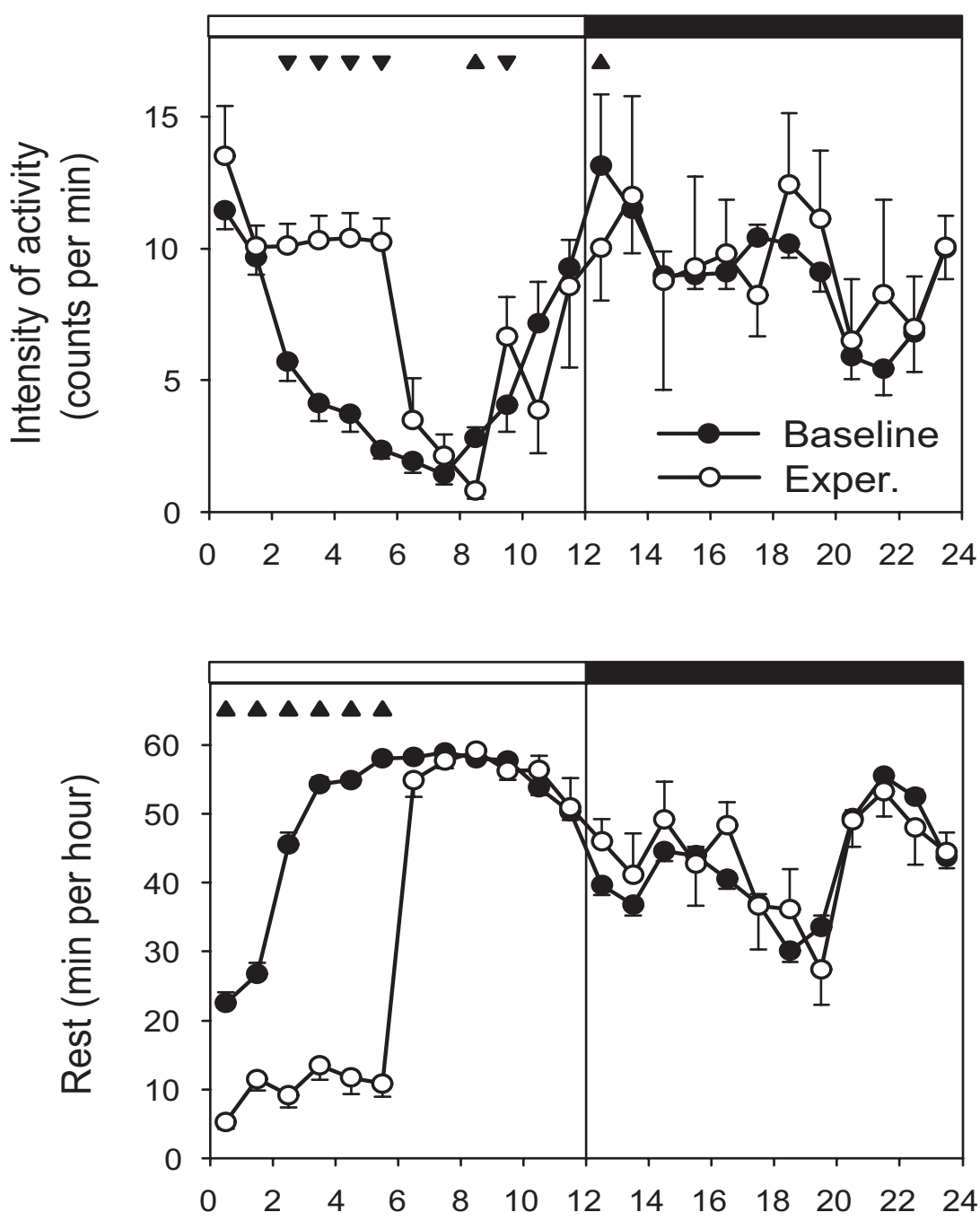

Delayed sleep deprivation
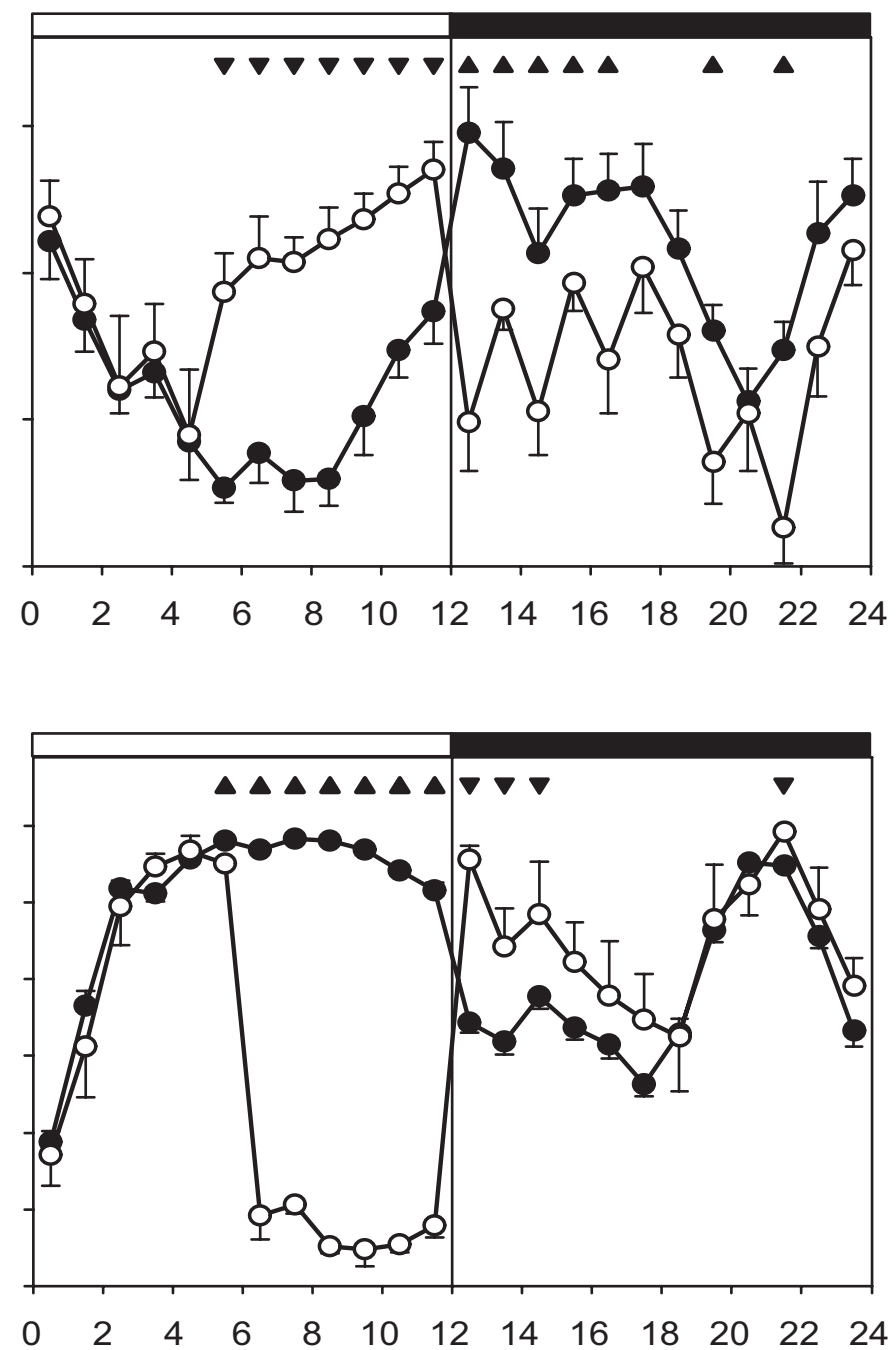

Time (hours) 
Figure 4

\section{Recovery after sleep deprivation}

Immediate sleep deprivation Delayed sleep deprivation
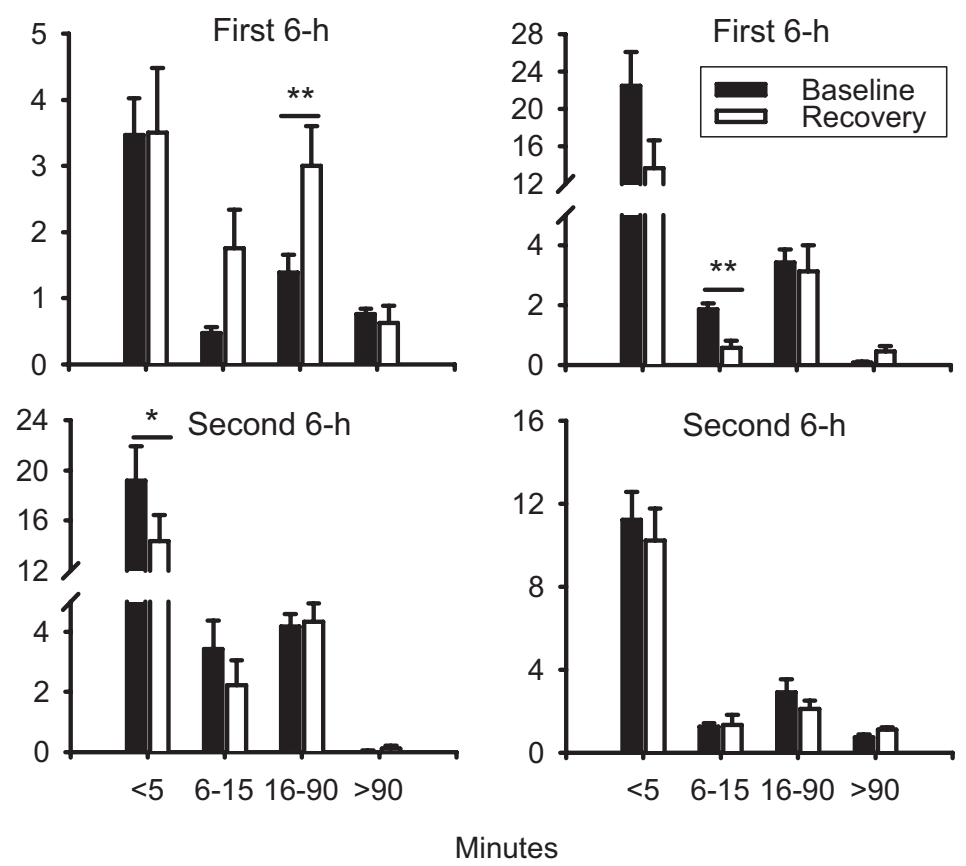

Minutes 
Figure 5

Corticosterone

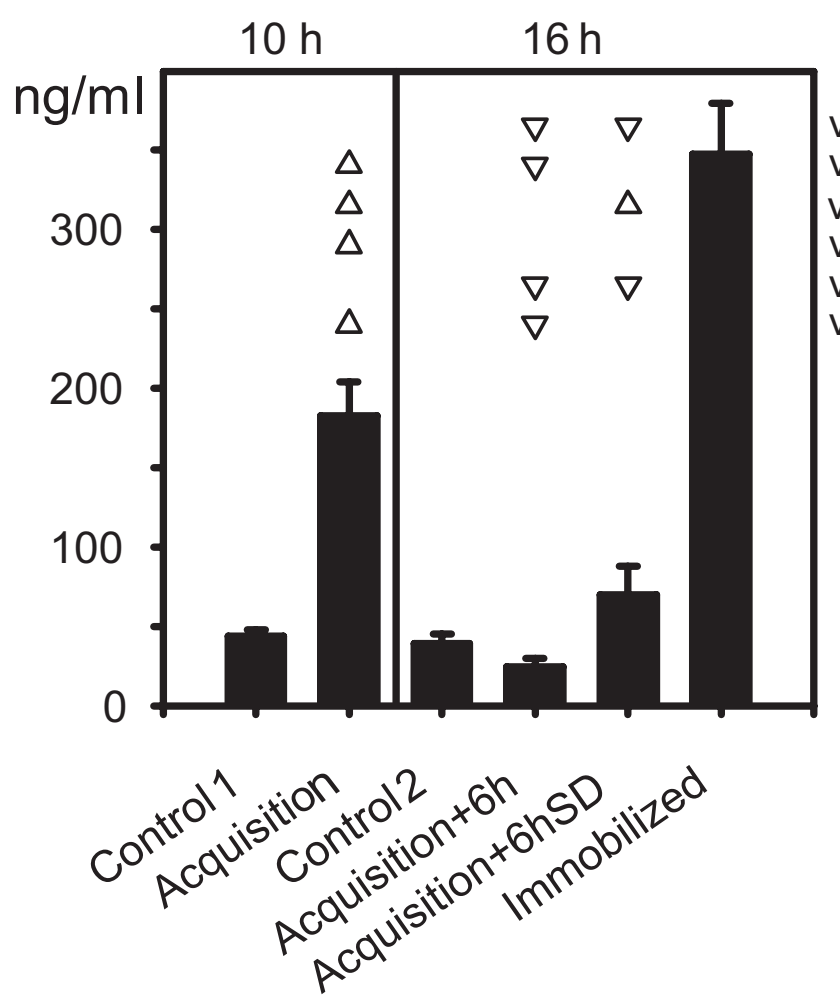

ACTH \begin{tabular}{l|ll|llll|l}
$\begin{array}{l}\text { vs Immobilized } \\
\text { v Acquisition+6hSD } \\
\text { vs Acquisition+6h }\end{array}$ & $\nabla$ & $\nabla$ & $\nabla$ & $\nabla$ & $\nabla$ & & $p g / m l$ \\
600
\end{tabular} vs Control2 vs Acquisition vs Control 1

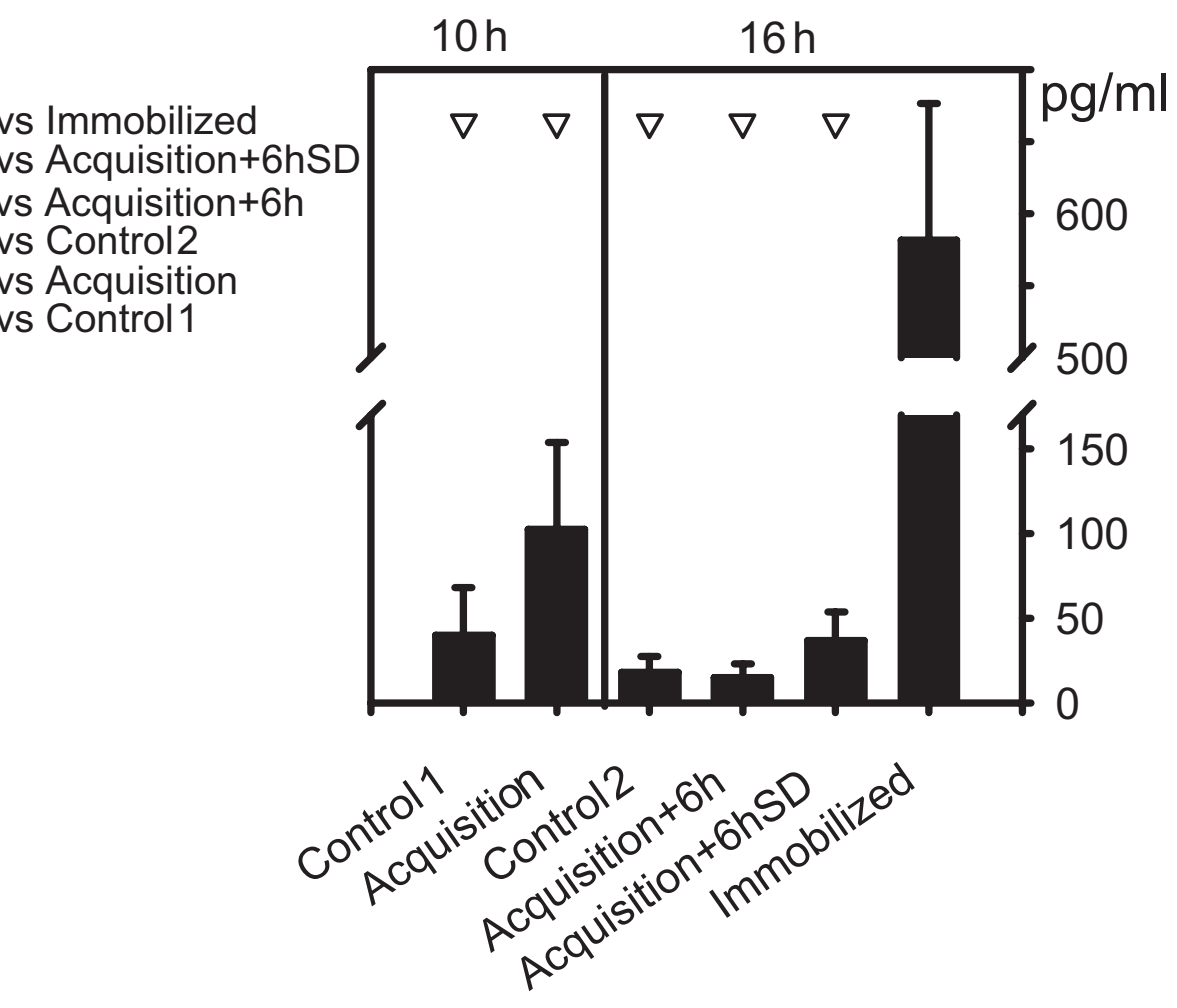

